\title{
Ecotoxicity Evaluation of Pristine and Indolicidin-coated Silver Nanoparticles in Aquatic and Terrestrial Ecosystem
}

This article was published in the following Dove Press journal:

International Journal of Nanomedicine

\author{
Annarita Falanga (D) \\ Antonietta Siciliano ${ }^{2}$ \\ Mariateresa Vitiello ${ }^{3}$ \\ Gianluigi Franci ${ }^{4}$ \\ Valentina Del Genio (iD ${ }^{5}$ \\ Stefania Galdiero $\mathbb{1 D}^{5}$ \\ Marco Guida $\mathbb{D}^{2}$ \\ Federica Carraturo $\mathbb{( D}^{2}$ \\ Amir Fahmi ${ }^{6, *}$ \\ Emilia Galdiero (iD ${ }^{2 *}$ \\ 'Department of Agricultural Science, \\ University of Naples Federico II, Portici \\ 80055, Italy; ${ }^{2}$ Department of Biology, \\ University of Naples Federico II, Naples \\ 80100 , Italy; ${ }^{3}$ Department of Clinical \\ Pathology, Virology Unit, "San Giovanni \\ di Dio e Ruggi d'Aragona Hospital", \\ Salerno, Italy; ${ }^{4}$ Department of Medicine, \\ Surgery and Dentistry "Scuola Medica \\ Salernitana," University of Salerno, \\ Baronissi, Italy; ${ }^{5}$ Department of \\ Pharmacy, School of Medicine, University \\ of Naples Federico II, Naples 80134, Italy; \\ ${ }^{6}$ Rhein-Waal University of Applied \\ Sciences, Kleve D-47533, Germany
}

*These authors contributed equally to this work

Correspondence: Emilia Galdiero; Amir Fahmi

Tel +39081679182

Email egaldier@unina.it; Amir. fahmi@hochschule-Rhein-Waal.de
Background: Metallic nanoparticles (NPs) are highly exploited in manufacturing and medical processes in a broad spectrum of industrial applications and in the academic sectors. Several studies have suggested that many metallic nanomaterials including those derived by silver (Ag) are entering the ecosystem to cause significant toxic consequences in cell culture and animal models. However, ecotoxicity studies are still receiving limited attention when designing functionalized and non.-functionalized AgNPs.

Objective: This study aimed to investigate different ecotoxicological profiles of AgNPs, which were analyzed in two different states: in pristine form uncoated AgNPs and coated AgNPs with the antimicrobial peptide indolicidin. These two types of AgNPs are exploited for a set of different tests using Daphnia magna and Raphidocelis subcapitata, which are representatives of two different levels of the aquatic trophic chain, and seeds of Lepidium sativum, Cucumis sativus and Lactuca sativa.

Results: Ecotoxicological studies showed that the most sensitive organism to AgNPs was crustacean $D$. magna, followed by $R$. subcapitata and plant seeds, while AgNPs coated with indolicidin (IndAgNPs) showed a dose-dependent decreased toxicity for all three.

Conclusion: The obtained results demonstrate that high ecotoxicity induced by AgNPs is strongly dependent on the surface chemistry, thus the presence of the antimicrobial peptide. This finding opens new avenues to design and fabricate the next generation of metallic nanoparticles to ensure the biosafety and risk of using engineered nanoparticles in consumer products.

Keywords: silver nanoparticles, antimicrobial peptide indolicidin, in vivo toxicity, phytotoxicity, nanotoxicology

\section{Introduction}

Bacterial diseases represent one of the biggest health issues in the world and we are now facing the significant challenge of antimicrobial resistance, which has determined the urgency to develop new therapeutics. Indeed, bacteria have evolved several resistance mechanisms against antibiotics, rendering the treatment of infectious diseases a major threat to public health. ${ }^{1}$

In this scenario, nanotechnology is emerging as a novel strategy to combat pathologies previously treated with conventional antibiotics. Among metal nanoparticles (NPs), silver nanoparticles (AgNPs) are considered potential candidates thanks to their broad spectrum antibacterial /antiviral properties and their cost

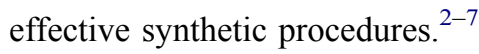


The combined use of silver NPs and other drugs seem to be promising; essentially, the nanosystem would be involved in multiple interactions attacking multiple targets in the same microbe and thus the microorganisms will be unable to develop resistance. ${ }^{8}$ Moreover, antimicrobial peptides (AMPs) constitute a class of evolutionarily conserved multifunctional molecules produced by the innate immune system of a variety of organisms which display a broad-spectrum activity, a low propensity to induce resistance $e^{9-11}$ and are outstanding molecules for clinical use. $^{12-14}$

Among AMPs, indolicidin is a small peptide rich in proline and tryptophan residues which has been previously used against severe acne and skin infections caused by methicillin resistant Staphylococcus aureus. ${ }^{15}$ We previously reported that AgNPs coated with indolicidin have a good antibacterial activity also with low doses of indolicidin attached to nanoparticles, lower than those used with indolicidin or NPs alone. ${ }^{16}$

Likewise, the opportunity to use AgNPs coated with indolicidin as a novel antimicrobial, determines the urgent need to better define their possible toxicological effects due to their potential release into the environment.

Furthermore, the production, use and marketing of AgNPs have rapidly increased in recent years and AgNPs are inevitably released into the environment with the result of creating significant concerns about their potential to cause negative effects on the environment and human health. ${ }^{17,18}$ AgNPs could reach the freshwater environment by human discharge because of their large application; thus, the potential environmental hazard has become a public concern. ${ }^{19}$ AgNPs could accumulate into different level organisms and there could be trophic transferred to food webs, which implies risks for human health. ${ }^{20}$ Moreover, some studies reported that the rate and amounts of AgNP dissolution both in biological and environmental media may depend on their physicochemical properties determined by the size, stabilization and presence of other molecules. $^{21-28}$

The AgNP toxicity has already been studied in aquatic organisms such as Daphnia magna, ${ }^{26,29-33}$ where toxicity is strongly dependent on the specific properties of the produced nanoparticles (size, capping, shape) and on their coating, which may modify their effect on living organisms; therefore, toxicity needs to be evaluated case by case on different models of aquatic organisms. ${ }^{34}$

For instance, some researchers showed that size is crucial. AgNPs have been shown to be more toxic than nanosize $\mathrm{Ag}$ spheres and wires in zebrafish embryos. ${ }^{35}$ The surface coating could affect the shape, aggregation and dissolution. Katawa et $\mathrm{al}^{36}$ showed that uncoated AgNPs had more cytotoxic effects and caused much stronger damage to chromosomes than polystyrene-AgNPs; Nguyen et $\mathrm{al}^{37}$ reported that uncoated AgNPs suppressed the inflammatory responses and enhanced oxidative stress in macrophage and epithelial cells more than PVP-coated AgNPs. However, toxicity changes due to alterations in the physiochemical properties was not well understood yet. $^{38}$

Generally, ecotoxicological studies use one or two bioindicators and analyze data obtained between a few hours and a few days without covering a period of months or more that is more realistic to study the whole ecosystem.

We previously analyzed the ecotoxicity of other metal nanoparticles. For instance, we ascertained that in spite of a decrement in toxicity of quantum dots (QDs) coated with indolicidin compared to QDs alone in multigenerational tests in D. magna, the complex at sublethal doses caused not only biophysical changes, but also an increase of stress response and metabolomic patterns. ${ }^{39,40}$ We also showed the effect of exposure of Saccharomyces cerevisiae to AgNPs functionalized and not with indolicidin ${ }^{41}$ and we found a link between oxidative stress, genotoxicity and gene expression of $Y C A 1$, with a decrease of all these parameters in AgNPs functionalized compared to AgNPs alone.

D. magna, a freshwater filter-feeding crustacean, is one of the most susceptible organisms used in ecotoxicity experiments and a standard test organism for EPA, OECD and ISO protocols. Moreover, since D. magna is at the bottom of the food chain in freshwater aquatic ecosystems, any modification in the quality or quantity of its population can lead to changes in the population of other aquatic organisms.

Previous findings indicated the sensitivity of Raphidocelis subcapitata, (also located at the bottom of the food chain) to various contaminants, ${ }^{42}$ and the importance of using the algae as a template for ecological risk. ${ }^{43}$

Furthermore, plants constitute a significant link in ecotoxicological studies, due to their ecological and economic importance; therefore, we performed a phytotoxicity test using seeds of Lepidium sativum, Cucumis sativus, and Lactuca sativa. Plant toxicity is of paramount importance since during the growth, plants absorb large amounts of essential and nonessential elements, which at certain 
concentrations may be toxic and can be transferred along the food chain to end-consumers, which are productive livestock and ultimately human beings. Moreover seed germination and root elongation tests are largely used for environmental biomonitoring. ${ }^{4,45}$ These methods were developed to assess the toxicity of polluted liquid samples on seed germination and root elongation. ${ }^{46,47}$

The proposed study aims to investigate the ecotoxicity and phytotoxicity of AgNPs, AgNPs coated with indolicidin (IndAgNPs), and indolicidin. To the best of our knowledge this is the first report of environmental assessment of potential toxicity due to accidental release of novel silver formulations where a notably antimicrobial peptide, indolicidin, is complexed with AgNPs and the selected organisms have niches that cover almost whole water ecosystems.

\section{Materials and Methods Chemicals and Reagents}

All chemicals were obtained from Sigma-Aldrich, Fluka (Buchs, Switzerland), or LabScan Stillorgan, Ireland) unless stated otherwise. Fmoc-protected amino acids, coupling reagents, and Rink amide p-methylbenzhydrylamine (MBHA) resin were purchased from CalbiochemNovabiochem (Laufelfingen, Switzerland).

\section{Peptide Synthesis}

The peptide indolicidin was synthesized on a scale of 100 $\mu$ mol using the standard solid-phase-9-fluorenylmethoxycarbonyl (Fmoc) method as previously reported. ${ }^{48}$ The crude peptide was cleaved from the resin and purified by RP-HPLC using a LC8 Shimadzu HPLC system (Shimadzu Corporation, Kyoto, Japan) equipped with a UV lambda-Max Model 481 detector; for purification, a Phenomenex (Torrance, CA, USA) $\mathrm{C}_{18}$ column was used and eluted with $\mathrm{H}_{2} \mathrm{O} / 0.1 \%$ TFA (A) and $\mathrm{CH}_{3} \mathrm{CN} / 0.1 \%$ TFA (B) from $20-80 \%$ over $20 \mathrm{~min}$ at a flow rate of $20 \mathrm{~mL} \mathrm{~min}{ }^{-1}$. The peptide was obtained with good yields (50-60\%), purity and identity were checked by analytical LC-MS analyses by using Finnigan Surveyor MSQ single quadrupole electrospray ionization (Finnigan/Thermo Electron Corporation San Jose, CA, USA).

\section{Preparation of Silver Colloids Using Hydrazine}

$150 \mu \mathrm{L}$ hydrazine monohydrate $\left(\mathrm{N}_{2} \mathrm{H}_{4} \cdot \mathrm{H}_{2} \mathrm{O}\right.$, Sigma Aldrich) were added to $1 \mathrm{~mL} \mathrm{AgNO}_{3}$ solution (1 mM).
Deionized water was added to the solution up to $2 \mathrm{~mL}$, mixed for one minute and allowed to settle at room temperature for four hours. To prepare silver colloids in the presence of indolicidin, $150 \mu \mathrm{L} \mathrm{N}_{2} \mathrm{H}_{4} \cdot \mathrm{H}_{2} \mathrm{O}$ were added to $1 \mathrm{~mL} \mathrm{AgNO}_{3}$ solution (1 mM). The solution was filled up to $2 \mathrm{~mL}$ with the peptide solution (indolicidin in deionized water $=560 \mu \mathrm{g} / \mathrm{mL}$ ), mixed and allowed to settle at room temperature. The nominal concentrations of the obtained solutions were $0.5 \mathrm{mM}$ of AgNPs alone and $0.5 \mathrm{mM}$ of AgNPs $+238 \mu \mathrm{g} / \mathrm{mL}$ of indolicidin for the complex. We reported the concentrations for all the subsequent experiments as a function of the concentration of the peptide indolicidin. When we used AgNPs without the peptide, we used the same amount of NPs contained in the relative solution with indolicidin (for simplicity of comparisons we indicate it with the concentration value of indolicidin).

\section{Characterization by UV-VIS}

$\mathrm{UV}$-Vis spectra of the resulting nanoparticle solutions were recorded at room temperature using a Lambda $25 \mathrm{UV}-\mathrm{Vis}$ spectrophotometer (Perkin Elmer). The monochromator slit width was $10 \mathrm{~nm}$.

\section{Characterization by TEM}

The morphology of AgNPs was analyzed using a Transmission Electron Microscope (TEM Jeol $2200 \mathrm{fs}$ ) operated at $200 \mathrm{keV}$. Prior to TEM measurements, $0.3 \mathrm{~mL}$ of each sample were spotted onto holey carbon film coated 200 mesh copper grids from quantifoil.

\section{Test Organisms and Culture Conditions}

D. magna daphnids were cultured in our laboratory in M4 medium $^{49}$ at a constant temperature of $20^{\circ} \mathrm{C}$ and a photoperiod of $16 \mathrm{~h}$ light and $8 \mathrm{~h}$ dark. Culture medium was changed twice a week and daphnids were fed with a suspension of green algae.

R. subcapitata algae was maintained in our laboratory, in $500 \mathrm{~mL}$ culture flasks with $200 \mathrm{~mL}$ of medium (ISO 982 2012) prepared by adding macro and micronutrient solutions to Milli-Q water. The $\mathrm{pH}$ of medium was 7.5. Algal cultures were grown under continuous illumination equipped by white fluorescent lamps at $25^{\circ} \mathrm{C}$ on an orbital shaker at $100 \mathrm{rpm}$. Cultures were maintained in exponential growth phase subcultivating a small amount of old culture in a fresh sterile medium every seven days. The plant species assayed were: L. sativum, C. sativus and L. sativa. The seeds were sterilized in $10 \% \mathrm{Na}-$ 
hypochlorite solution for $20 \mathrm{~min}$ to avoid fungal growth and washed with distilled water many times.

\section{Daphnia Acute Toxicity Test}

It was carried out according to OECD guideline 202. Every test was done four times with four control groups. Briefly, 30 neonates aged $<24$ hwere divided into four groups and exposed to different concentrations of AgNPs, IndAgNPs, indolicidin with a range $0.0005 \mu \mathrm{g} / \mathrm{L}$ to $0.016 \mu \mathrm{g} / \mathrm{L}$.

All tests were performed in a water bath system with a constant temperature $\left(20 \pm 2^{\circ} \mathrm{C}\right)$ and $16 \mathrm{~h} \mathrm{light} / 8 \mathrm{~h}$ dark cycles and animals were not fed during the experiments. After $48 \mathrm{~h}$ exposure, neonates that were unable to swim within 15 seconds were considered to be immobilized after gentle agitation. GraphPad Prism program was used to create dose-response curves with a nonlinear regression model and calculate the median effect concentration $\mathrm{EC}_{50}$ and $\mathrm{EC}_{20}$ values, as well as their confidence intervals (95\%). After 24 and $48 \mathrm{~h}$ of exposure, the immobilization and mortality of the $D$. magna were estimated using a light microscope (Leica EZ4HD, 10×/20) and any visible uptake and adsorption of nanoparticles by D. magna were photographed with a digital camera. Furthermore, the live Daphnia were classified in four groups according to their swimming type: normal swimming (NOR), erratic swimming (ERR), at the bottom (BOT), at the surface (SUR) ${ }^{50}$

\section{Daphnia Chronic Test}

The chronic toxicity test was performed according to the standard protocol OECD Guideline 202. ${ }^{51}$ Neonates aged $<24 \mathrm{~h}$ were exposed to a sublethal concentration of AgNPs, IndAgNPs and indolicidin $(1 \mu \mathrm{g} / \mathrm{L})$ for a period of 21 days. The exposure conditions were the same as those used in the routine cultures. Neonates were fed every two days and media and nanoparticle solution were changed simultaneously. Survival, offspring and newly born neonates were observed and monitored every day. At the end of the exposure, surviving parents and newborns were collected and photographed. To determine significant differences in the survival organisms like total number of neonates, total number of clutches, age at first reproduction at the end of the experiment, we used a oneway ANOVA.

\section{Algae Growth Inhibition Test}

The growth inhibition test of $R$. subcapitata was assessed following the method of OECD 201. ${ }^{52}$ We used exponentially growing algal cultures that were exposed to various concentrations of the test substances under controlled conditions.

Briefly, with an initial concentration of $5 \dot{\times} 10^{4}$ cells $/ \mathrm{mL}$ was incubated in each well containing serial dilutions (starting from the concentration of $10 \mu \mathrm{g} / \mathrm{L}$ with arithmetic progression of 1:2) of AgNPs and IndAgNPs and indolicidin on MBL medium. For each sample, eight concentrations in a geometric series were tested in the concentration range previously settled in a preliminary test. All assays were replicated three times. Plates were incubated in a light-temperature controlled chamber at $25^{\circ} \mathrm{C}$ for 72 $\mathrm{h}$ with a photoperiod of $16 \mathrm{~h}: 8 \mathrm{~h}$ light-dark. Samples from each well were read in a spectrophotometer at 670 $\mathrm{nm}$ after $72 \mathrm{~h}$. Cell growth inhibition was the endpoint measured after $72 \mathrm{~h}$ in a Bürker cell counting chamber and calculated by dividing the difference of the number of control and sample cells to the number of control cells. The specific growth rate of $R$. subcapitata in each replicate culture was calculated from the logarithmic increase in cell density in the intervals from 0 to $72 \mathrm{~h}$ using the following equation:

$$
\mu=\frac{\ln N_{n}-\ln N_{0}}{t_{n}-t_{0}}
$$

where $N_{0}$ is the cell concentration at $t=0, N_{\mathrm{n}}$ the final cell concentration after $72 \mathrm{~h}$ of exposure, $t_{0}$ the time of start measurement, and $t_{\mathrm{n}}$ the time of last measurement (hours from start). The inhibition of the cell growth, expressed as percentage, at sample (\% I) was calculated as the difference between the rate growth of the control and the rate growth, Values are expressed as the mean $\pm \mathrm{SD}$ of the replicates $(p \leq 0.05)$.

$\mathrm{EC}_{50}$ and $\mathrm{EC}_{20}$ were calculated using GraphPad Prism program and Excel macro REGTOX.

\section{Phytotoxicity Test}

Phytotoxicity assays were conducted following the OECD 208 method. ${ }^{53}$ Seeds were sown in $90 \mathrm{~mm}$ diameter petri dishes that contained one sheet of Whatman No. 1 filter paper as support and $3 \mathrm{~mL}$ of test solutions. Ten seeds, in three replicates, were sown per container. The seeds were incubated in a growth chamber at $25^{\circ} \mathrm{C}$ for $72 \mathrm{~h}$. After this period, the germination index (GI) was calculated by multiplying the germinated seed number (G) and length of roots (L). The toxic effect was expressed as percentage germination index (GI\%) with respect to the control and calculated as follows: GI $\%=(\mathrm{IGc}-\mathrm{IGs}) / \mathrm{GIc} * 100$, where 
IGs and IGc are the germination indices calculated for samples and control, respectively.

\section{Results}

\section{IndAgNPs Synthesis and Characterization}

AgNPs were prepared via an in situ approach by applying redox reactions. The AMP indolicidin was used to coat AgNPs; in particular, indolicidin was used as a capping agent, hydrazine was added as a reducing agent to donate electrons in order to allow Ag ions to complete their outer valance shell and be converted into Ag nanoparticles. The functional groups of the AMP indolicidin possess high affinity to transition elements and are useful to anchor peptide chains on the particle surface. This facilitated passivation mechanism also allows us to avoid the Ag cluster aggregation. In principle, the generated $\mathrm{Ag}$ atoms formed by redox reactions tend to coalesce and grow into larger clusters. Nonetheless, by adding the indolicidin molecules, this coalescent process is inhibited and the peptide acts not only as a cluster stabilizer but also contributes to control the size and size distribution of the generated AgNPs.

UV-Vis spectrometer was used to confirm the formation of AgNPs in colloidal solution already reported in a previous work. ${ }^{16}$ The different sizes of the Ag nanoclusters were determined by TEM (Figure 1). The particles were nearly spherical in shape and evenly distributed in size with an average dimension of $10-40 \mathrm{~nm}$ if they were uncoated or coated.
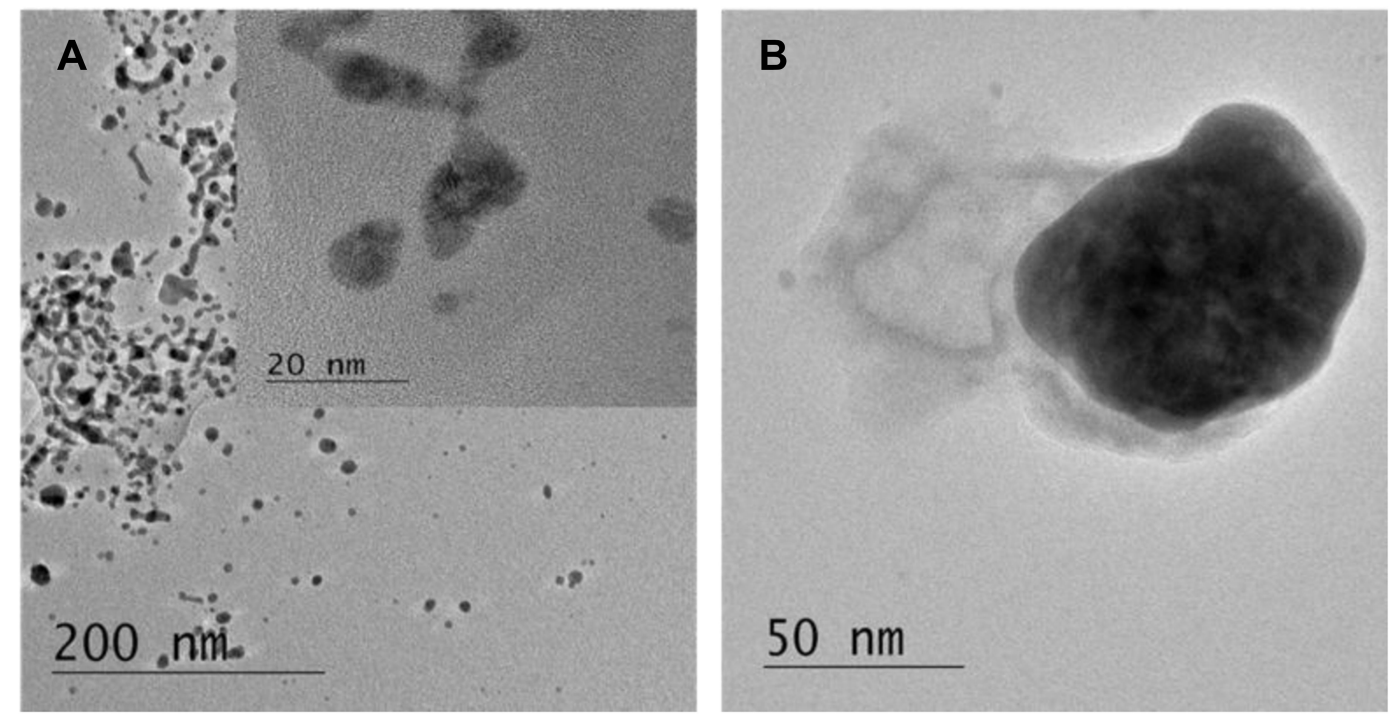

Figure I TEM micrographs of colloidal silver nanoparticles with: (I) indolicidin and (2) without indolicidin TEM micrographs in. Figure I show the size and the morphologies of AgNPs pristine (uncoated) and Ag NPs templated within indolicidin matrixes. (A) Demonstrates that the morphologies of generated AgNPs passivated with the indolicidin chains are nearly spherical with defined particle size around $15 \mathrm{~nm}$. In contrast, the AgNPs generated without adding the indolicidin molecules are significantly larger in size within the range of $40 \mathrm{~nm}$ as shown in Figure 2B. 
A

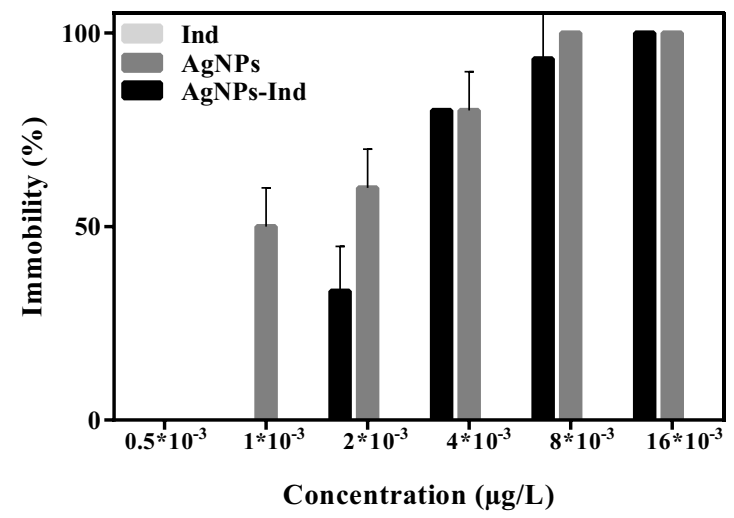

B

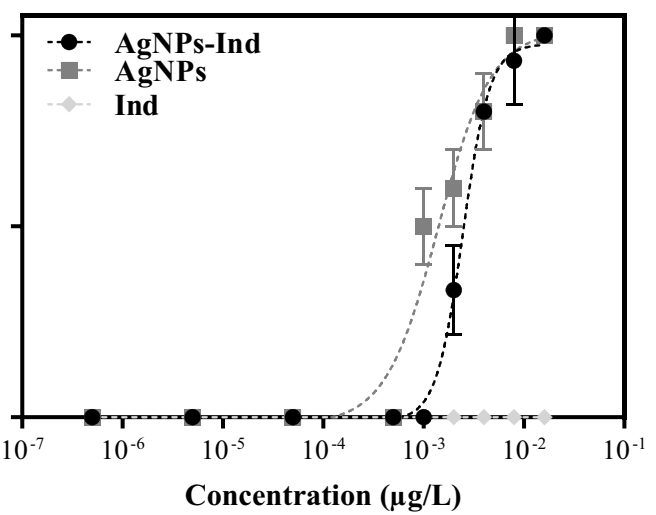

Figure 2 Immobilization comparison of D. magna exposed to different concentrations of indolicidin, AgNPs and AgNPs with indolicidin after $48 \mathrm{~h}$ (A) and fitted curves by $\log 10$ in $48 \mathrm{~h}(\mathbf{B})$.

In some cases, the ingestion of NPs also prevented the movement of Daphnia. In fact, we evaluated the normal and abnormal swimming of the live Daphnia as shown in Table 2. We noticed that while in the control groups, Daphnia exhibited a normal swimming, in the samples with AgNPs and IndAgNPs, it showed three different kind of swimming BOT (bottom), SUR (surface), ERR (erratic).

Again, the percentage of abnormalities was dosedependent and most of them were observed in groups treated with AgNPs. In the group exposed only to indolicidin, at the same doses, we noticed that even if $100 \%$ Daphnia were mobile, at higher concentration of indolicidin their swimming was abnormal (Table 2). In fact, $90 \%$ of daphnids had a bottom swimming and $10 \%$ an erratic swimming. Daphnids exposed to AgNPs, showed a high percentage of normal swimming only at $0.5 \times 10^{-3}$ concentration tested while IndAgNPs showed the result at both concentrations $0.5 \times 10^{-3} \mu \mathrm{g} / \mathrm{L}-16 \times 10^{-3} \mu \mathrm{g} / \mathrm{L}$.

The toxicity of AgNPs, IndAgNPs and indolicidin changed in the chronic experiment with $D$. magna where a sublethal doses of $1 \mu \mathrm{g} / \mathrm{L}$ for 21 days was used.
Beginning from day 13, the survival for AgNPs and IndAgNPs was $80 \%$ and remained constant until day 19 . After day 19, the survival decreased to $70 \%$ until the end of the test for AgNPs exposure, as shown in Figure 4A.

Daphnia exposed to sublethal dose of AgNPs, IndAgNPs and indolicidin showed a significant delay of reproduction times compared to controls as shown in Figure 4B. In fact the first brood was observed after 16 days in the group of D. magna exposed to AgNPs and after 14 days in the group exposed to IndAgNPs. Daphnia treated with the peptide indolicidin alone reproduced significantly earlier, so that the first brood was detected at day 10 .

The number of offspring and the average number of neonates differed between control Daphnia not exposed and those exposed to sublethal concentration of AgNPs and IndAgNPs (Figure 5B).

When we compared silver bioaccumulation at the end of exposure, we noticed that daphnids exposed to AgNPs and IndAgNPs showed amounts of nanoparticles in the digestive tract, droplets usually surrounding the

Table I Nominal Concentration Values on D.magna of $\mathrm{EC}_{50}$ and $\mathrm{EC}_{20}$ of AgNPs and AgNPs Coated with Indolicidin, with Their $95 \%$ $\mathrm{Cl}, \mathrm{r}$-Square Value $\left(\mathrm{R}^{2}\right)$ and Degrees of Freedom $(\mathrm{df})$

\begin{tabular}{|l|l|l|l|l|l|}
\hline & Indolicidin & EC $_{\mathbf{5 0}}(\mathbf{C l})$ & EC $_{\mathbf{2 0}}(\mathbf{C l})$ & $\mathbf{R}^{\mathbf{2}}$ & $\mathbf{d f}^{-}$ \\
\cline { 3 - 7 } & & ND & ND & ND & ND \\
\hline \multirow{2}{*}{ D. magna } & AgNPs & $1.8(1.7-1.9)$ & $0.9(0.8-1.1)$ & 0.99 & 25 \\
& IndAgNPs & $2.9(2.7-3.1)$ & $1.9(1.8-2.1)$ & 0.98 & 25 \\
\hline \multirow{2}{*}{ R. subcapitata } & Indolicidin & ND & $1.7(1.08-2.6)$ & 0.99 & 6 \\
& AgNPs & $0.95(0.4-2.1)$ & $0.2(0.07-0.43)$ & 0.89 & 6 \\
& IndAgNPs & $2.9(1.8-4.7)$ & $0.3(0.16-0.44)$ & 0.97 & 6 \\
\hline
\end{tabular}

Abbreviation: ND, not determined. 


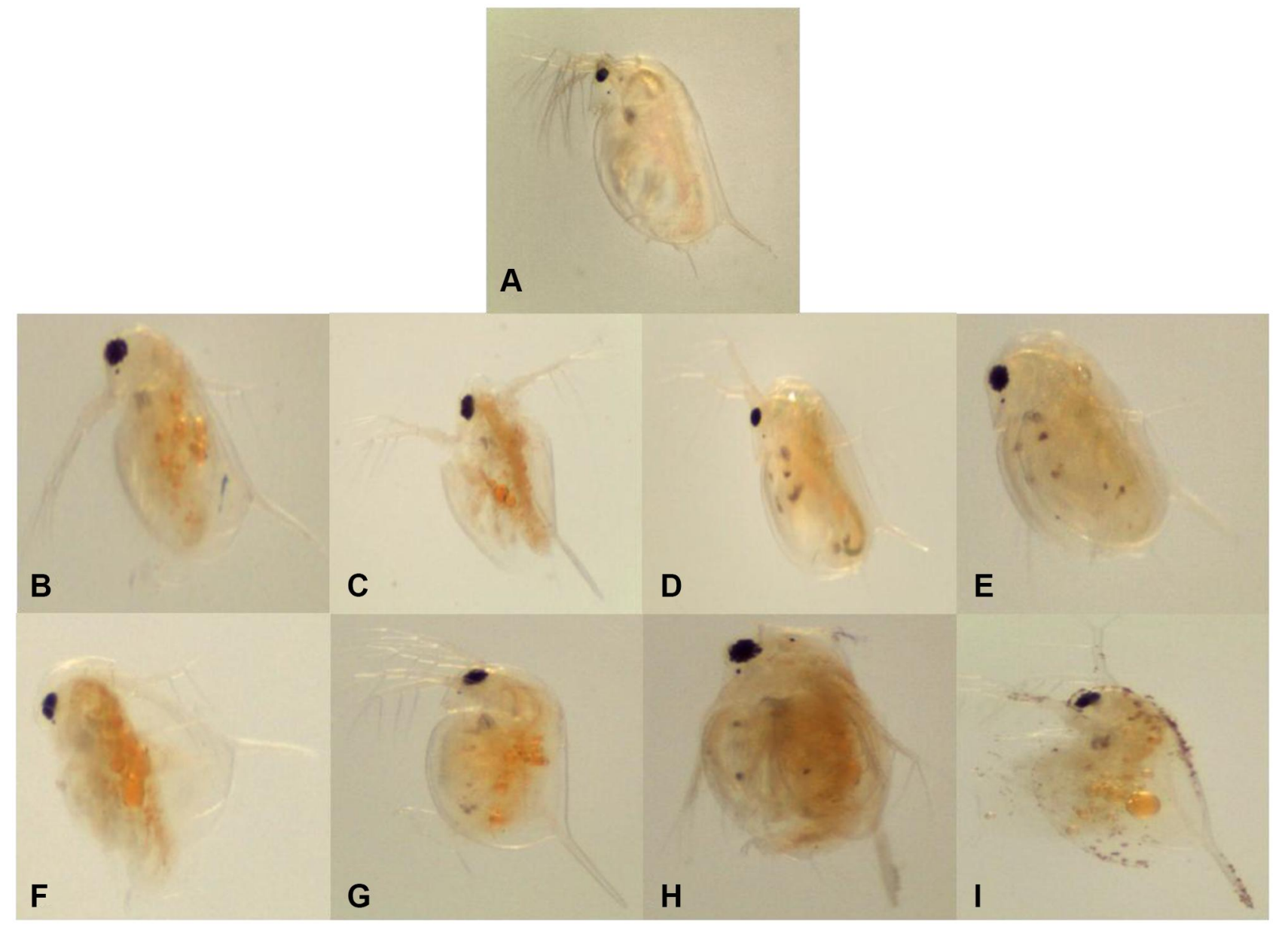

Figure 3 Light microscope images of D. magna after $48 \mathrm{~h}$ of exposure. Figure shows daphnids from control group (A) and daphnids subjected at $2 \mu \mathrm{g} / \mathrm{L}(\mathbf{B}), 4 \mu \mathrm{g} / \mathrm{L}(\mathbf{C}), 8 \mu \mathrm{g} / \mathrm{L}$ (D) and $16 \mu \mathrm{g} / \mathrm{L}(\mathrm{E})$ of IndAgNPs and at 2 (F), 4 (G), 8 (H) and (I) $16 \mu \mathrm{g} / \mathrm{L}$ of AgNPs.

intestine and clogging of the carapace (Figure 4). No sign of impaired feeding and no effects on growth were observed.

\section{Toxicity Tests on $R$. subcapitata}

$R$. subcapitata has been chosen because microalgae have an important role in the aquatic system and are a member of food chains in the aquatic environment; moreover, they have a short generation time and respond quickly to environmental changes. We tested the growth inhibition at $72 \mathrm{~h}$. Nominal concentration-based dose-response curves for AgNPs, IndAgNPs, and indolicidin are shown in Figure 5A and B.

AgNPs exposure, caused a dose-response increase in growth inhibition, reaching $78 \%$ at the concentration of 5 $\mu \mathrm{g} / \mathrm{L}$, which was considered toxic.

The same trend was observed for IndAgNPs even if the percentages were lower compared to pristine AgNPs. Indolicidin showed a growth inhibition of $25 \%$ at the concentration of $16.10-3 \mu \mathrm{g} / \mathrm{L}$.
The $\mathrm{EC}_{50}$ and $\mathrm{EC} 20$ with $95 \% \mathrm{CI}$ were 2.9 and $0.3 ; 0.95$ and 0.2 not determined and 1.7, respectively for IndAgNPs, AgNPs, indolicidin (Table 1). These results confirm that after $72 \mathrm{~h}$ exposure, it is sufficient only $0.3 \mu \mathrm{g} / \mathrm{L}$ of AgNPS to inhibit $50 \%$ of the population growth. This result may have important ecological consequences if we consider that $R$. subcapitata was grown under ideal conditions without the stressors that are likely found in nature together with other components with synergistic effects.

\section{Effect of Nanoparticles on Seed Germination}

Seed germination and root growth inhibitory tests, which are highly sensitive, simple to perform and inexpensive, were used to determine the phytotoxic effects of nanoparticles.

When growing, plants absorb large amounts of essential and nonessential elements, which at certain concentration may be toxic and can be transferred along the food 
Table 2 Percentage of Normal and Abnormal D. magna During Swimming $48 \mathrm{~h}$ Exposure to Different Concentration of AgNPs, AgNPs Coated with Indolicidin and Indolicidin

\begin{tabular}{|c|c|c|c|c|c|c|}
\hline Sample & Conc. $(\mu \mathrm{g} / \mathrm{L})$ & Normal \% & Abnormal \% & Bottom \% & Surface \% & \% Erratic \\
\hline \multirow[t]{6}{*}{ Indolicidin } & $0.5 \times 10^{-3}$ & 86.6 & 13.4 & 13.4 & 0 & 0 \\
\hline & $1 \times 10^{-3}$ & 60 & 40 & 20 & 0 & 20 \\
\hline & $2 \times 10^{-3}$ & 0 & 100 & 80 & 6.6 & 13.4 \\
\hline & $4 \times 10^{-3}$ & 0 & 100 & 80 & 6.6 & 13.4 \\
\hline & $8 \times 10^{-3}$ & 0 & 100 & 80 & 6.6 & 13.4 \\
\hline & $16 \times 10^{-3}$ & 0 & 100 & 90 & 0 & 10 \\
\hline \multirow{6}{*}{ AgNPs } & $0.5 \times 10^{-3}$ & 86.6 & 13.4 & 13.4 & 0 & 0 \\
\hline & $1 \times 10^{-3}$ & 0 & 100 & 44.4 & 11.2 & 33.4 \\
\hline & $2 \times 10^{-3}$ & 0 & 100 & 57.3 & 14.2 & 28.5 \\
\hline & $4 \times 10^{-3}$ & 0 & 100 & 80 & 0 & 20 \\
\hline & $8 \times 10^{-3}$ & - & - & - & - & - \\
\hline & $16 \times 10^{-3}$ & - & - & - & - & - \\
\hline \multirow[t]{6}{*}{ IndAgNPs } & $0.5 \times 10^{-3}$ & 86.6 & 13.4 & 13.4 & 0 & 0 \\
\hline & $1 \times 10^{-3}$ & 66.6 & 33.4 & 20 & 0 & 13.4 \\
\hline & $2 \times 10^{-3}$ & 0 & 100 & 80 & 0 & 20 \\
\hline & $4 \times 10^{-3}$ & 0 & 100 & 100 & 0 & 0 \\
\hline & $8 \times 10^{-3}$ & 0 & 100 & 100 & 0 & 0 \\
\hline & $16 \times 10^{-3}$ & - & - & - & - & - \\
\hline
\end{tabular}

chain to consumers. L. sativum, C. sativus, L. sativa are considered important models to test phytotoxicity. Various concentrations ranging from $1 \mu \mathrm{g} / \mathrm{L}$ to $5 \mu \mathrm{g} / \mathrm{L}$ were analyzed for the effects on seedling growth. Exposure of L. sativum, to different doses of AgNPs, IndAgNPs, indolicidin showed a low inhibition of germination with IG between 51, 29 and 76.5\%. Furthermore, the exposition of C. sativus showed a low inhibition at the highest concentration tested for all substances. Only L. sativa showed differences of inhibition. Indolicidin caused a low inhibition showing an IG of $47.8 \%$ and $65.5 \%$, while for both AgNPs and IndAgNPs showed a marked inhibition of growth with value IG lower than $40 \%$ (Figure $6 \mathrm{~A}-\mathrm{C}$ ). We noticed that we have a bioaccumulation of NPs only for L. sativa exposed to AgNPs and an abnormal growth of roots in all three bioindicators exposed to AgNPs compared to negative control but also in samples exposed to AgNPs with indolicidin (not shown). We speculate that the seed germination increase may be due to water uptake by seeds as previously reported. ${ }^{55}$ This activated water uptake process could be responsible for the significantly faster germination rates and higher biomass production for the plants that were exposed to small sized nanoparticles even if molecular mechanisms that induce water uptake inside plant seeds are not clear and require further investigation.

\section{Discussion}

The toxicity of AgNPs to aquatic species is well documented in the literature but different toxicity results could be obtained from nanoparticles prepared using different methods and above all functionalized by different coatings; ${ }^{56}$ thus, the toxicity of any new material needs to be investigated case by case.

D. magna is an indicator organism for effects on the primary aquatic consumers that eat algae and are, in turn, consumed by organisms higher in the food chain. As a consequence, any response found in Daphnia could have consequences for populations of other aquatic organisms.

Zhao and Wang ${ }^{57}$ showed that more than $70 \%$ of AgNPs accumulated in the daphnids was due to ingestion of silver adsorbed by algae. Our results highlight the importance of AgNP transport over the food chain as they could not be entirely depurated from the daphnids. It has been shown that the noticed toxicity of AgNPs to D. magna and algae was the results of both $\mathrm{Ag}^{+}$ions and nanosilver. ${ }^{58}$

The mechanisms of AgNP toxicity in terrestrial plants is even more unclear. Plants constitute a significant link in ecotoxicological studies, due to their ecological and economic importance. The bioaccumulation of nanoparticles in the aquatic ecosystem may unfavorably affect the productivity of primary producers such as algae that 

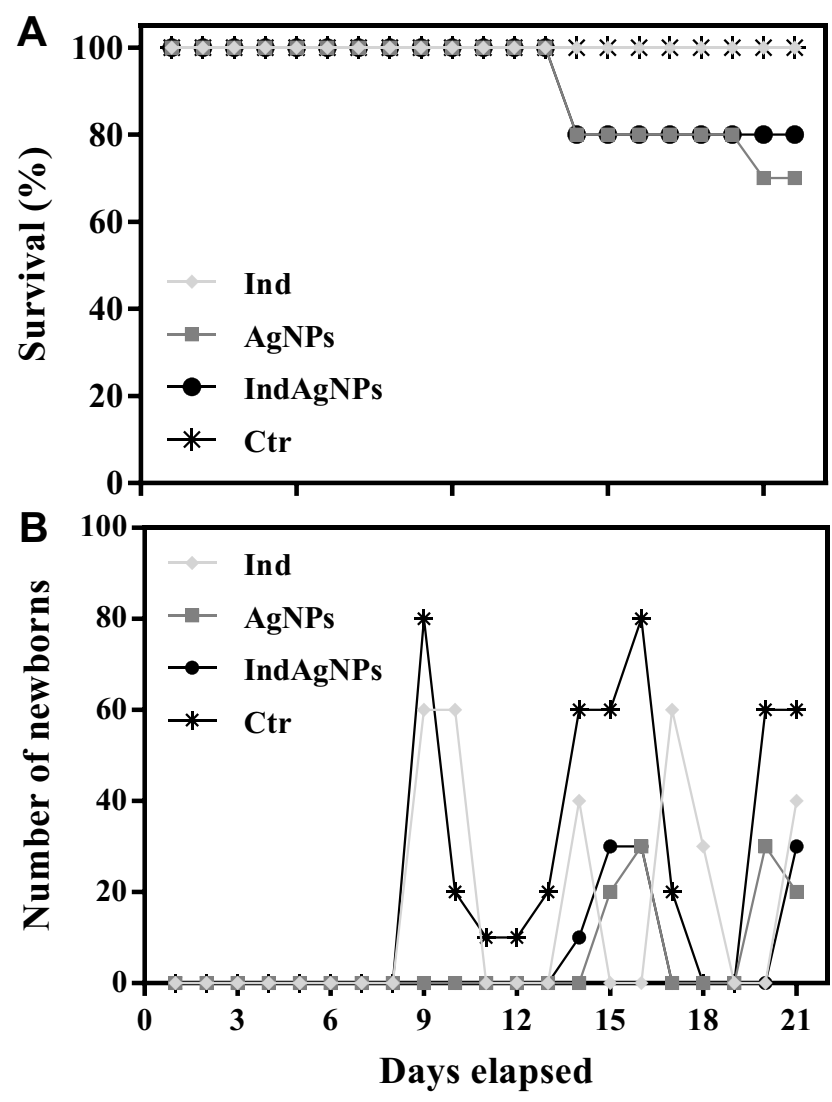

Figure 4 Cumulative percentage survival for each of the tested substances subjected to a chronic text for 21 days (A) Cumulative live offspring produced per female for each of the tested substances after 21 days of exposure (B).

might eventually bring about environmental disorders leading to the alteration of the delicate balance between the structure and function of the ecosystem. In this study, $R$. subcapitata was found to be greatly influenced by the AgNPs compared to IndAgNPs at increasing con centrations.
The $\mathrm{EC}_{50}$ values obtained for AgNPs, IndAgNPs and indolicidin indicate that the most sensitive organism to AgNPs was crustacean D. magna followed by R. subcapitata and plant seeds. This order of sensitivity is in agreement with the literature. ${ }^{59}$

The present study demonstrates that AgNPs produce acute toxicity on $D$. magna at a concentration of $1.6 \mu \mathrm{g} / \mathrm{L}$; nonetheless, only daphnids exposed to the highest concentrations showed brown color in their gut lines, indicating that AgNPs were ingested.

In the chronic toxicity tests with a sublethal concentration of $1 \mu \mathrm{g} / \mathrm{L}$, daphnids showed a reduced reproduction and offspring delay even if the mortality was low because of the sublethal concentration used. Even lower mortality was found for AgNPs functionalized with indolicidin. The delay of offspring as well as the reduction of neonates in the chronic test with D. magna can be considered a response to the toxic effect of AgNPs. The comparison of the results obtained for the chronic and acute toxicity against daphnids did not produce significant variation in the $\mathrm{EC}_{10}$, as far as it concerns the reproductive rate, the probability of survival to maturation and doses; it is likely that the amount of dissolved silver decreased in the presence of food algae.

The acute toxic effects of AgNPs, IndAgNPs, and indolicidin on seeds at various concentrations were determined by seed germination and root elongation inhibition tests. The exposition of seeds to AgNPs, IndAgNPs, and indolicidin had a clear phytotoxic effect only for AgNPs and on L. sativa and was dependent upon the concentration of AgNPs. The low seed germination rate and low root elongation associated with AgNPs are likely related to

A

B
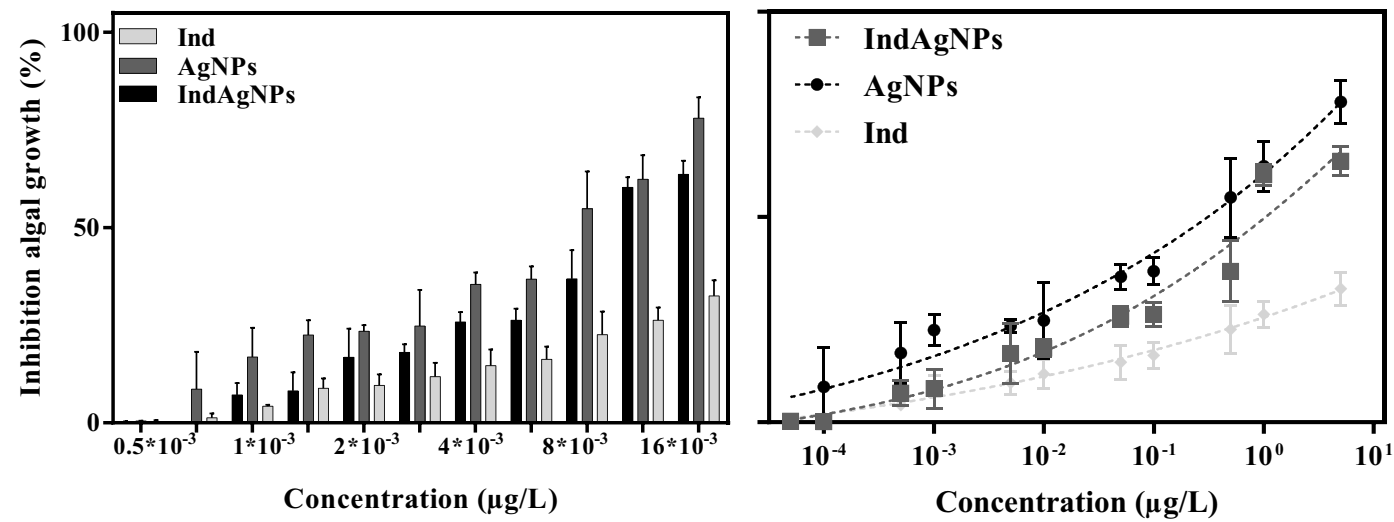

Figure 5 Growth inhibition of the microalgae R. subcapitata after $72 \mathrm{~h}$ exposure to AG, IndAg, Ind. Column representation to easily compare values (A), row representation to show the inhibition increase as a function of concentration (B). 

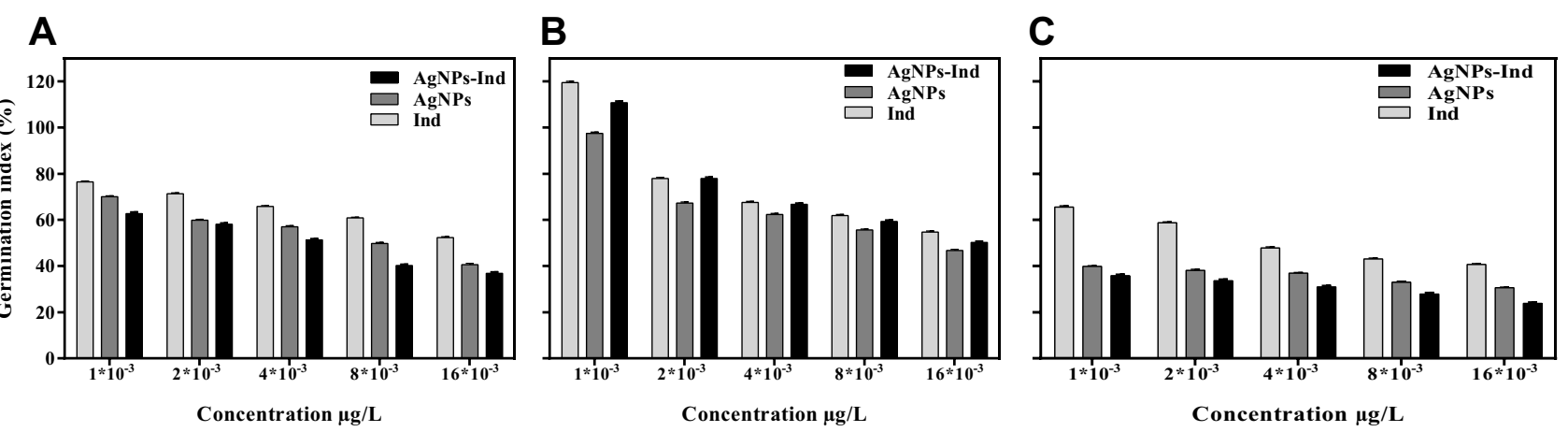

Figure 6 Effect of 72 h-seed germination to different concentration of AgNPs, AgNPs-Ind, Ind for L. sativum (A), C. sativus (B), L. sativa (C).

metal ions released from the nanoparticle tested. It is reported in literature that small size AgNPs present more severe toxicity to aquatic organisms, which was attributed to the higher dissolution rate and extent. Surface coatings utilized to maintain the stability of AgNPs can reduce $\mathrm{Ag}^{+}$ release depending on the type of coating. For instance, Polyvinylpyrrolidone (PVP) AgNPs have been found to release more ions compared to citrate AgNPs, likely because $\mathrm{Ag}^{+}$can bind to carboxylic groups, and citrate may act as a reducing agent to hinder the surface oxidation of AgNPs. Thus, PVP-AgNPs are likely to be more prone to $\mathrm{Ag}^{+}$release compared to Cit-Ag NPs. At the same time, these surface coatings strongly affect the stability of NPs in the order PVP-AgNPs $>$ Cit-AgNPs and the toxicity of Cit-AgNPs is higher than the toxicity of PVP-AgNPs indicating that aggregation is also a key parameter and is influenced by the coating. Our NPs were prepared in presence of hydrazine which both helps the stabilization and likely reduces the $\mathrm{Ag}^{+}$release; the addition of the peptide coating further seems to reduce toxicity and may represent a valuable strategy for the development of novel antimicrobial drugs with lower toxicity to the environment. ${ }^{35}$ The use of NPs has significant advantages in research and medical applications, but at the same time it is required the acquisition of toxicity data to ensure the biosafety. The major finding of our research, based on the data of the three indicator species, is that AgNPs coated with an AMP such as indolicidin are less toxic for the environment compared to naked AgNPs. These conclusions should be reevaluated using other endpoints or testing other nanomaterials containing silver.

\section{Acknowledgments}

The authors would like to thanks Dr M. Heidelmann at ICAN University of Duisburg-Essen for conducting the
TEM measurements. We would like to acknowledge Dr Christine Strehmel for her fruitful support.

\section{Disclosure}

The authors report no conflicts of interest in this work.

\section{References}

1. Davies J, Davies D. Origins and evolution of antibiotic resistance. Microbiol Mol Biol Rev. 2010;74(3):417-433. doi:10.1128/ MMBR.00016-10

2. Dos Santos CA, Seckler MM, Ingle AP, et al. Silver nanoparticles: therapeutical uses, toxicity, and safety issues. J Pharm Sci. 2014;103 (7):1931-1944. doi:10.1002/jps.24001

3. Gaikwad S, Ingle A, Gade A, et al. Antiviral activity of mycosynthesized silver nanoparticles against herpes simplex virus and human parainfluenza virus type 3. Int J Nanomedicine. 2013;8:4303-4314.

4. Galdiero S, Falanga A, Vitiello M, et al. Silver nanoparticles as potential antiviral agents. Molecules. 2011;16(10):8894-8918. doi:10.3390/molecules 16108894

5. Rai M, Deshmukh SD, Ingle AP, et al. Metal nanoparticles: the protective nanoshield against virus infection. Crit Rev Microbiol. 2014.

6. Rai M, Kon K, Ingle A, et al. Broad-spectrum bioactivities of silver nanoparticles: the emerging trends and future prospects. Appl Microbiol Biotechnol. 2014;98(5):1951-1961. doi:10.1007/s00253013-5473-x

7. Rai MK, Deshmukh SD, Ingle AP, et al. Silver nanoparticles: the powerful nanoweapon against multidrug-resistant bacteria. $J$ Appl Microbiol. 2012;112(5):841-852. doi:10.1111/j.1365-2672.2012.05 253.x

8. Seil JT, Webster TJ. Antimicrobial applications of nanotechnology: methods and literature. Int J Nanomedicine. 2012;7:2767-2781.

9. Hancock REW, Sahl H-G. Antimicrobial and host-defense peptides as new anti-infective therapeutic strategies. Nat Biotech. 2006;24 (12):1551-1557. doi:10.1038/nbt1267

10. Marr AK, Gooderham WJ, Hancock RE. Antibacterial peptides for therapeutic use: obstacles and realistic outlook. Curr Opin Pharmacol. 2006;6(5):468-472. doi:10.1016/j.coph.2006.04.006

11. Galdiero S, Falanga A, Berisio R, et al. Antimicrobial peptides as an opportunity against bacterial diseases. Curr Med Chem. 2015;22 (14):1665-1677. doi:10.2174/0929867322666150311145632

12. Gordon YJ, Romanowski EG, McDermott AM. A review of antimicrobial peptides and their therapeutic potential as anti-infective drugs. Curr Eye Res. 2005;30(7):505-515. doi:10.1080/0271368059096 8637 
13. Lazarev VN, Govorun VM. Antimicrobial peptides and their use in medicine. Appl Biochem Microbiol. 2010;46(9):803-814. doi:10.11 34/S0003683810090012

14. Ghosh C, Sarkar P, Issa R, et al. Alternatives to conventional antibiotics in the era of antimicrobial resistance. Trends Microbiol. 2019;27(4):323-338. doi:10.1016/j.tim.2018.12.010

15. Melo MN, Dugourd D, Castanho MA. Omiganan pentahydrochloride in the front line of clinical applications of antimicrobial peptides. Recent Pat Antiinfect Drug Discov. 2006;1(2):201-207. doi:10.2174/ 157489106777452638

16. Zannella C, Vitiello M, Falanga A, et al. Antibacterial activity of indolicidin-coated silver nanoparticles in oral disease. Appl Sci. 2020;10(5):1837.

17. Handy RD, Owen R, Valsami-Jones E. The ecotoxicology of nanoparticles and nanomaterials: current status, knowledge gaps, challenges, and future needs. Ecotoxicology. 2008;17(5):315-325. doi:10.1007/s10646-008-0206-0

18. Savolainen K, Alenius H, Norppa H, et al. Risk assessment of engineered nanomaterials and nanotechnologies - a review. Toxicology. 2010;269(2-3):92-104. doi:10.1016/j.tox.2010.01.013

19. Du J, Tang J, Xu S, et al. A review on silver nanoparticles-induced ecotoxicity and the underlying toxicity mechanisms. Regul Toxicol Pharmacol. 2018;98:231-239. doi:10.1016/j.yrtph.2018.08.003

20. Lekamge S, Miranda AF, Ball AS, et al. The toxicity of coated silver nanoparticles to Daphnia carinata and trophic transfer from alga Raphidocelis subcapitata. PLoS One. 2019;14(4):e0214398e0214398. doi:10.1371/journal.pone.0214398

21. Courtois P, Rorat A, Lemiere S, et al. Ecotoxicology of silver nanoparticles and their derivatives introduced in soil with or without sewage sludge: a review of effects on microorganisms, plants and animals. Environ Pollut. 2019;253:578-598. doi:10.1016/j.envpol.2019.07.053

22. Aruoja V, Dubourguier H-C, Kasemets K, et al. Toxicity of nanoparticles of $\mathrm{CuO}, \mathrm{ZnO}$ and $\mathrm{TiO} 2$ to microalgae Pseudokirchneriella subcapitata. Sci Total Environ. 2009;407(4):1461-1468. doi:10.1016/j.scitotenv.2008.10.053

23. Farkas J, Christian P, Gallego-Urrea JA, et al. Uptake and effects of manufactured silver nanoparticles in rainbow trout (Oncorhynchus mykiss) gill cells. Aquat Toxicol. 2011;101(1):117-125.

24. Sinha R, Karan R, Sinha A, et al. Interaction and nanotoxic effect of $\mathrm{ZnO}$ and $\mathrm{Ag}$ nanoparticles on mesophilic and halophilic bacterial cells. Bioresour Technol. 2011;102(2):1516-1520. doi:10.1016/j. biortech.2010.07.117

25. Adams LK, Lyon DY, Alvarez PJJ. Comparative eco-toxicity of nanoscale $\mathrm{TiO} 2, \mathrm{SiO} 2$, and $\mathrm{ZnO}$ water suspensions. Water Res. 2006;40(19):3527-3532. doi:10.1016/j.watres.2006.08.004

26. Allen HJ, Impellitteri CA, Macke DA, et al. Effects from filtration, capping agents, and presence/absence of food on the toxicity of silver nanoparticles to Daphnia magna. Environ Toxicol Chem. 2010;29 (12):2742-2750. doi:10.1002/etc.329

27. Oberdörster E, Zhu S, Blickley TM, et al. Ecotoxicology of carbon-based engineered nanoparticles: effects of fullerene (C60) on aquatic organisms. Carbon. 2006;44(6):1112-1120. doi:10.1016/j. carbon.2005.11.008

28. Galdiero E, Maselli V, Falanga A, et al. Integrated analysis of the ecotoxicological and genotoxic effects of the antimicrobial peptide melittin on Daphnia magna and Pseudokirchneriella subcapitata. Environ Pollut. 2015;203:145-152. doi:10.1016/j.envpol.20 15.03 .046

29. Gaiser BK, Biswas A, Rosenkranz P, et al. Effects of silver and cerium dioxide micro- and nano-sized particles on Daphnia magna. J Environ Monit. 2011;13(5):1227-1235. doi:10.1039/c1em10060b

30. Griffitt RJ, Luo J, Gao J, et al. Effects of particle composition and species on toxicity of metallic nanomaterials in aquatic organisms. Environ Toxicol Chem. 2008;27(9):1972-1978. doi: $10.1897 / 08-002.1$
31. Kennedy AJ, Hull MS, Bednar AJ, et al. Fractionating nanosilver: importance for determining toxicity to aquatic test organisms. Environ Sci Technol. 2010;44(24):9571-9577. doi:10.1021/es1025382

32. Sakka Y, Skjolding LM, Mackevica A, et al. Behavior and chronic toxicity of two differently stabilized silver nanoparticles to Daphnia magna. Aquat Toxicol. 2016;177:526-535. doi:10.1016/j.aquatox.2016.06.025

33. Pem B, Pongrac IM, Ulm L, et al. Toxicity and safety study of silver and gold nanoparticles functionalized with cysteine and glutathione. Beilstein J Nanotechnol. 2019;10:1802-1817. doi:10.3762/ bjnano.10.175

34. Freixa A, Acuña V, Sanchís J, et al. Ecotoxicological effects of carbon based nanomaterials in aquatic organisms. Sci Total Environ. 2018;619-620:328-337. doi:10.1016/j.scitotenv.2017.11.095

35. Zhang W, Ke S, Sun C, et al. Fate and toxicity of silver nanoparticles in freshwater from laboratory to realistic environments: a review. Environ Sci Pollut Res Int. 2019;26(8):7390-7404. doi:10.1007/ s11356-019-04150-0

36. Kawata K, Osawa M, Okabe S. In vitro toxicity of silver nanoparticles at noncytotoxic doses to HepG2 human hepatoma cells. Environ Sci Technol. 2009;43(15):6046-6051. doi:10.1021/es900754q

37. Nguyen KC, Seligy VL, Massarsky A, et al. Comparison of toxicity of uncoated and coated silver nanoparticles. J Phys Conf Ser. 2013;429:012025. doi:10.1088/1742-6596/429/1/012025

38. Mishra P, Vinayagam S, Duraisamy K, et al. Distinctive impact of polystyrene nano-spherules as an emergent pollutant toward the environment. Environ Sci Pollut Res Int. 2019;26(2):1537-1547. doi:10.1007/s11356-018-3698-z

39. Falanga A, Mercurio FA, Siciliano A, et al. Metabolomic and oxidative effects of quantum dots-indolicidin on three generations of Daphnia magna. Aquat Toxicol. 2018;198:158-164. doi:10.1016/j. aquatox.2018.03.001

40. Maselli V, Siciliano A, Giorgio A, et al. Multigenerational effects and DNA alterations of QDs-indolicidin on Daphnia magna. Environ Pollut. 2017;224:597-605. doi:10.1016/j.envpol.2017.02.043

41. de Alteriis E, Falanga A, Galdiero S, et al. Genotoxicity of gold nanoparticles functionalized with indolicidin towards Saccharomyces cerevisiae. J Environ Sci. 2018;66:138-145.

42. de Paiva Magalhaes D, da Costa Marques MR, Fernandes Baptista D, et al. Selecting a sensitive battery of bioassays to detect toxic effects of metals in effluents. Ecotoxicol Environ Saf. 2014;110:73-81. doi:10.1016/j.ecoenv.2014.08.019

43. Samei M, Sarrafzadeh M-H, Faramarzi MA. The impact of morphology and size of zinc oxide nanoparticles on its toxicity to the freshwater microalga, Raphidocelis subcapitata. Environ Sci Pollut Res Int. 2019;26(3):2409-2420. doi:10.1007/s11356-018-3787-z

44. Wang W, Williams JM. The use of phytotoxicity tests (common duckweed, cabbage, and millet) for determining effluent toxicity. Environ Monit Assess. 1990;14(1):45-58. doi:10.1007/BF00394356

45. Wang W, Keturi P. Comparative seed germination tests using ten plant species for toxicity assessment of a metal engraving effluent sample. Water Air Soil Pollut. 1990;52(3-4):369-376. doi:10.1007/ BF00229444

46. Araujo A, Sahyoun F, Monteiro R. Evaluation of toxicity of textile sludge compost on seed germination and root elongation of soybean and wheat. Ecossistema. 2001;26(2):117-119.

47. Siddiqi KS, Husen A. Plant response to engineered metal oxide nanoparticles. Nanoscale Res Lett. 2017;12(1):92.

48. Cantisani M, Leone M, Mignogna E, et al. Structure-activity relations of myxinidin, an antibacterial peptide derived from the epidermal mucus of hagfish. Antimicrob Agents Chemother. 2013;57 (11):5665-5673. doi:10.1128/AAC.01341-13

49. Elendt B-P, Bias W-R. Trace nutrient deficiency in Daphnia magna cultured in standard medium for toxicity testing. Effects of the optimization of culture conditions on life history parameters of D. magna. Water Res. 1990;24(9):1157-1167. doi:10.1016/00431354(90)90180-E 
50. Asghari S, Johari SA, Lee JH, et al. Toxicity of various silver nanoparticles compared to silver ions in Daphnia magna. J Nanobiotechnology. 2012;10(1):14. doi:10.1186/1477-3155-10-14

51. OECD. OECD Guidelines for Testing of Chemicals, Test No.202: Daphnia Sp., Acute Immobilization Test; 2004.

52. OECD. OECD Guidelines for Testing of Chemicals, Test No. 201: Freshwater Alga and Cyanobacteria, Growth Inhibition Test; 2006.

53. OECD. Terrestrial Plant: Growth Test OECD. Guideline for Chemicals. Paris: No., 208.Organization for Economic Cooperation and Development; 1984.

54. Ratte HT. Bioaccumulation and toxicity of silver compounds: a review. Environ Toxicol Chem. 1999;18(1):89-108. doi:10.1002/ etc. 5620180112

55. Khodakovskaya M, Dervishi E, Mahmood M, et al. Carbon nanotubes are able to penetrate plant seed coat and dramatically affect seed germination and plant growth. ACS Nano. 2009;3 (10):3221-3227. doi:10.1021/nn900887m
56. Maurer-Jones MA, Gunsolus IL, Murphy CJ, et al. Toxicity of engineered nanoparticles in the environment. Anal Chem. 2013;85 (6):3036-3049. doi:10.1021/ac303636s

57. Zhao CM, Wang WX. Biokinetic uptake and efflux of silver nanoparticles in Daphnia magna. Environ Sci Technol. 2010;44 (19):7699-7704. doi:10.1021/es101484s

58. Khoshnamvand M, Hao Z, Fadare OO, et al. Toxicity of biosynthesized silver nanoparticles to aquatic organisms of different trophic levels. Chemosphere. 2020;258:127346. doi:10.1016/j. chemosphere.2020.127346

59. Ivask A, Kurvet I, Kasemets K, et al. Size-dependent toxicity of silver nanoparticles to bacteria, yeast, algae, crustaceans and mammalian cells in vitro. PLoS One. 2014;9(7):e102108. doi:10.1371/ journal.pone.0102108

\section{Publish your work in this journal}

The International Journal of Nanomedicine is an international, peerreviewed journal focusing on the application of nanotechnology in diagnostics, therapeutics, and drug delivery systems throughout the biomedical field. This journal is indexed on PubMed Central, MedLine, CAS, SciSearch ${ }^{\mathbb{R}}$, Current Contents ${ }^{\mathbb{R}} /$ Clinical Medicine, $^{-}$
Journal Citation Reports/Science Edition, EMBase, Scopus and the Elsevier Bibliographic databases. The manuscript management system is completely online and includes a very quick and fair peer-review system, which is all easy to use. Visit http://www.dovepress.com/ testimonials.php to read real quotes from published authors. 